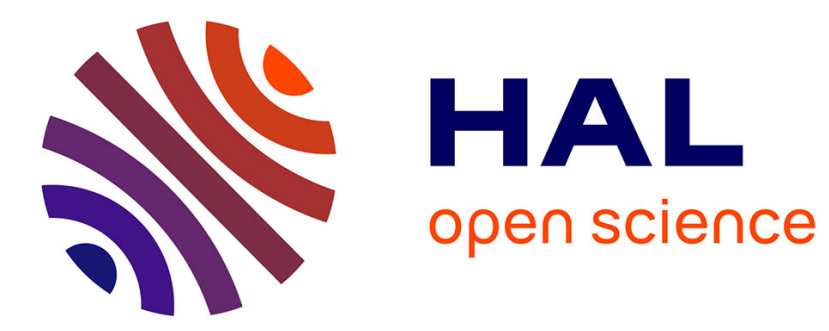

\title{
MAXIM, un macrosimulateur rapide de circuits
}

S. Dusausay, B. Gerbault, P. Philippe, J.F. Pône, G. Raynaud, R. Castagné

\section{To cite this version:}

S. Dusausay, B. Gerbault, P. Philippe, J.F. Pône, G. Raynaud, et al.. MAXIM, un macrosimulateur rapide de circuits. Revue de Physique Appliquée, 1987, 22 (11), pp.1529-1537. 10.1051/rphysap:0198700220110152900 . jpa-00245705

\section{HAL Id: jpa-00245705 https://hal.science/jpa-00245705}

Submitted on 1 Jan 1987

HAL is a multi-disciplinary open access archive for the deposit and dissemination of scientific research documents, whether they are published or not. The documents may come from teaching and research institutions in France or abroad, or from public or private research centers.
L'archive ouverte pluridisciplinaire HAL, est destinée au dépôt et à la diffusion de documents scientifiques de niveau recherche, publiés ou non, émanant des établissements d'enseignement et de recherche français ou étrangers, des laboratoires publics ou privés. 


\title{
MAXIM, un macrosimulateur rapide de circuits $\left({ }^{1}\right)$
}

\author{
S. Dusausay, B. Gerbault, P. Philippe (*), J. F. Pône, G. Raynaud (*) et R. Castagné \\ Institut d'Electronique Fondamentale, CNRS UA 22, Université Paris XI, 91405 Orsay Cedex, France \\ (*) Ecole Polytechnique Féminine, 3 bis rue Lakanal, 92330 Sceaux, France
}

(Reçu le 16 janvier 1987, révisé le 30 juin 1987, accepté le 3 juillet 1987)

\begin{abstract}
Résumé. - MAXIM est un macrosimulateur de circuits électriques permettant l'accès aux formes d'ondes des signaux. Il utilise une méthode nodale de mise en équation du circuit, décrit par macromodèles ou/et par composants élémentaires. La résolution se fait au moyen d'une méthode implicite du premier ordre (OSR), certains composants utilisent une résolution matricielle locale. MAXIM est utilisable sur tout ordinateur disposant d'un compilateur FORTRAN 77. Son processeur d'entrée permet un mode conversationnel. Son processeur de sortie permet la visualisation graphique des résultats sur de nombreux types de terminaux. MAXIM permet de simuler des circuits logiques et analogiques MESFET's et bipolaire hétérojonction GaAs et MOSFET's et bipolaire Silicium. De par son principe de calcul, les exemples de simulation montrent que MAXIM est capable de simuler des circuits de complexité importante en des temps de calculs réduits.
\end{abstract}

Abstract. - MAXIM is a macrosimulator for electrical circuits which gives acces to signal waveforms. Circuit, described using macromodels and/or elementary components, are decomposed using a nodal method. Circuit equations are solved using a first order implicit method (OSR), a local matricial method being used for some components. MAXIM can be used on every computer where a FORTRAN 77 compiler is available. Its input processor allows a conversational mode, and its output processor allows graphic outputs to be generated on a lot of terminals. MAXIM can be used to simulate logic and anologic MESFET's and heterojunction bipolar transistors GaAs, as well as MOSFET's and Silicon bipolar circuits. Due to the employed method, simulation examples show that MAXIM is able to simulate circuits having an important complexity, with small computing times.

\section{Introduction.}

L'accès aux formes d'ondes précises des signaux est une nécessité dans le cadre de la conception de circuits intégrés rapides.

Les simulateurs électriques classiques ne répondent en général pas à ce besoin pour des circuits de complexité moyenne, à cause d'un temps de calcul et d'une occupation mémoire trop importants.

MAXIM [1] est un logiciel qui répond à ces problèmes ; son processeur d'analyse permet d'obtenir des résultats en un temps de calcul réduit (fonction sub-linéaire du nombre de composants du circuit), et son organisation des données un encombrement faible de la mémoire (allocation dynamique de mémoire); son utilisation est facilitée par un langage d'entrée simple et interactif.

$\left.{ }^{1}\right)$ Etude partiellement financée par le CNET (convention $\mathrm{n}^{\circ}$ 85.8B.025.LAB).
MAXIM était initialement dédié à la simulation de circuits logiques et en utilisait les propriétés :

- répétitivité d'éléments de base,

- unidirectionnalité de ces éléments,

- latence : non-activité d'une partie du circuit.

En fait, l'évolution de MAXIM montre que toutes ces conditions ne sont pas nécessaires, en particulier l'unidirectionnalité des blocs fonctionnels n'est plus une condition indispensable au bon fonctionnement du simulateur. Cette possibilité de blocs multidirectionnels ouvre à MAXIM la voie de la simulation mixte c'est-à-dire de circuits découpés en macromodèles complexes connus du simulateur (portes logiques, amplificateur...), et en composants élémentaires (transistors, résistances, diodes, condensateurs). Une conséquence directe de cette possibilité est la simulation de circuits analogiques et de circuits comportant des transistors bipolaires, fortement bidirectionnels. 
Les phénomènes de propagation peuvent être pris en compte par MAXIM sous forme de retards purs appliqués aux signaux ; MACPRO [2], logiciel dont le principe repose sur les mêmes hypothèses et le même algorithme de base que MAXIM est spécialisé pour la simulation de ces phénomènes (réflexions, couplages etc.).

\section{Algorithme et méthode de calcul.}

MAXIM est basé sur la décomposition du circuit en macromodèles. Le processeur d'initialisation construit un graphe ordonné selon le sens préférentiel de passage de l'information dans le circuit.

A chaque valeur du temps (le pas sur temps est défini par l'utilisateur), les blocs sont analysés successivement en suivant le graphe ordonné, et des itérations sont éventuellement effectuées (au choix de l'utilisateur) pour améliorer la précision du résultat (méthode implicite de prédiction-correction [3]). Le calcul en chaque nœud et à chaque itération se fait par application de la relation suivante :

$$
V_{(t n+1)}=V_{(t n)}+\int_{t n}^{t n+1} I / C \mathrm{~d} u
$$

$V$ : tension au noud,

$I$ : somme algébrique des courants au nœud,

$C$ : capacité au nœud par rapport à la masse

La méthode de résolution est donc une méthode dite OSR (One Step Relaxation) utilisée notamment par les simulateurs ELDO [4] et REVE [5]. La structure logicielle et l'algorithme de calcul sont résumés figure 1.

Les plus importantes difficultés de cette analyse nodale résident dans le calcul des capacités équiva-

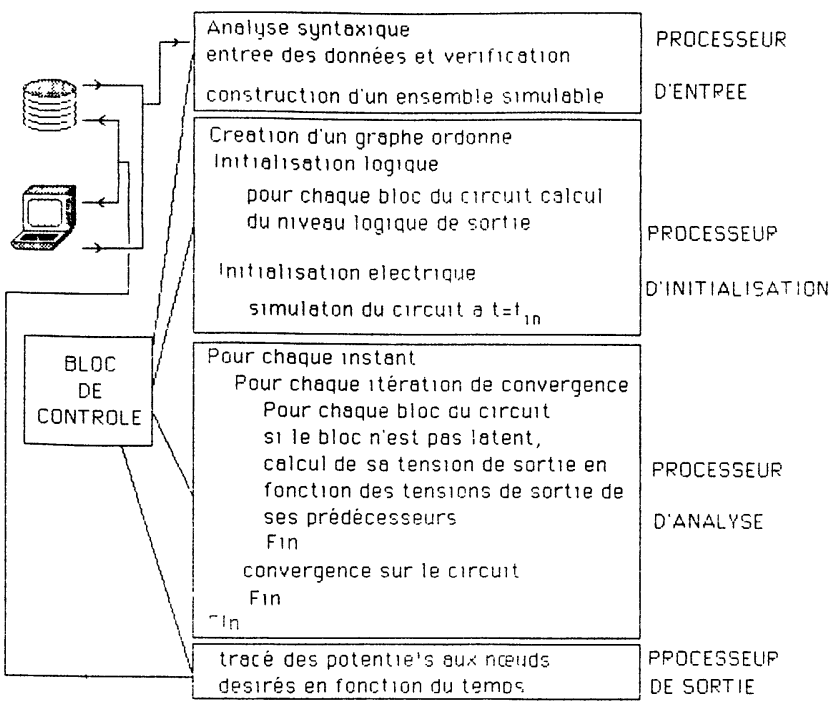

Fig. 1. - Structure logicielle et algorithme de calcul.

[Software structure and algorithm.] lentes des blocs fonctionnels en chaque nœud, et dans le découplage entre les entrées et les sorties. En effet, tout macromodèle décrit dans MAXIM doit se réduire à un ensemble de dipoles générateur de courant en parallèle sur un condensateur ayant un point à la masse, un dipole pour chaque entrée et un pour la sortie du bloc.

Deux techniques sont actuellement utilisées par MAXIM pour ramener les circuits sous cette forme ; un composant peut être représenté par des sources de courant et des capacités (une résistance étant équivalente à une source de courant contrôlée par une tension). La structure générale est alors celle de la figure $2 a$.

(a)

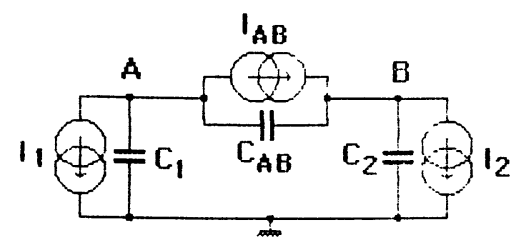

(b)

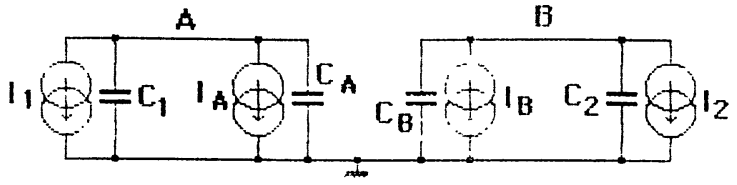

Fig. 2. - (a) structure générale, (b) même circuit après transformation.

[(a) general structure, (b) same circuit after transformation.]

Première technique: Utilisation du théorème de Miller.

Pour les nœuds $\mathrm{A}$ et B, les expressions des courants sont :

$$
\begin{aligned}
I_{1}=-I_{\mathrm{AB}}-C_{\mathrm{AB}}\left(\mathrm{d} V_{\mathrm{A}} / \mathrm{d} t-\mathrm{d} V_{\mathrm{B}} / \mathrm{d} t\right) & - \\
& -C_{1} \mathrm{~d} V_{\mathrm{A}} / \mathrm{d} t \\
I_{2}=+I_{\mathrm{AB}}+C_{\mathrm{AB}}\left(\mathrm{d} V_{\mathrm{A}} / \mathrm{d} t-\mathrm{d} V_{\mathrm{B}} / \mathrm{d} t\right) & - \\
& -C_{2} \mathrm{~d} V_{\mathrm{A}} / \mathrm{d} t .
\end{aligned}
$$

En utilisant le théorème de Miller, on peut ramener le circuit à celui de la figure $2 b$ ou :

$$
\begin{array}{ll}
C_{\mathrm{A}}=C_{\mathrm{AB}}\left(1-\mathrm{d} V_{\mathrm{B}} / \mathrm{d} V_{A}\right) & I_{\mathrm{A}}=I_{\mathrm{AB}} \\
C_{\mathrm{B}}=C_{\mathrm{AB}}\left(1-\mathrm{d} V_{\mathrm{A}} / \mathrm{d} V_{\mathrm{B}}\right) & I_{\mathrm{B}}=-I_{\mathrm{AB}} .
\end{array}
$$

Les quantités $\mathrm{d} V_{i} / \mathrm{d} V_{j}$ sont calculées à chaque pas de temps et à chaque itération.

Deuxième technique : Application du théorème de Norton.

La capacité $C_{\mathrm{AB}}$ et le courant $I_{\mathrm{AB}}$ sont ramenés aux nœuds $A$ et $B$ en calculant le générateur de Norton équivalent en ces deux nœuds. Ce théorème 
est applicable dans la mesure où l'on considère que les générateurs de courants ne sont pas liés pendant la durée d'un pas sur le temps (Fig. 2b) :

$$
\begin{aligned}
C_{\mathrm{A}} & =C_{\mathrm{AB}} C_{2} /\left(C_{\mathrm{AB}}+C_{2}\right) \\
I_{\mathrm{A}} & =\left(I_{2}-I_{\mathrm{AB}}\right) *\left(C_{\mathrm{AB}} /\left(C_{\mathrm{AB}}+C_{2}\right)\right)+I_{\mathrm{AB}} \\
C_{\mathrm{B}} & =C_{\mathrm{AB}} C_{1} /\left(C_{\mathrm{AB}}+C_{1}\right) \\
I_{\mathrm{B}} & =\left(I_{1}-I_{\mathrm{AB}}\right) *\left(C_{\mathrm{AB}} /\left(C_{\mathrm{AB}}+C_{1}\right)\right)-I_{\mathrm{AB}} .
\end{aligned}
$$

Il est à noter que dans ce calcul, les capacités $C_{1}$ et $C_{2}$ et les courants $I_{1}$ et $I_{2}$ tiennent compte de l'environnement du bloc (successeurs et prédécesseurs dans le graphe ordonné, et blocs connectés en parallèle sur les entrées).

Dans ces deux cas, des itérations de convergence réduisent l'effet du découplage temporel ; cet algorithme permet une bonne stabilité du calcul et des gains de temps de simulation importants par rapport à des simulateurs utilisant une méthode classique de résolution (méthode nodale ou du tableau tel SPICE [6] ou ASTEC [7].

\section{Portabilité.}

Pour permettre sa portabilité aisée sur de nombreux ordinateurs, un logiciel utilitaire est associé à MAXIM.

Cet utilitaire traduit les fichiers sources de MAXIM qui sont décrits dans un méta-langage, en FORTRAN 77 en tenant compte des particularités de l'ordinateur du site d'installation (Fig. 3).

Actuellement, MAXIM est implantable sur VAX 11/780/750 VMS, série SPERRY 1100, série PERKIN-ELMER 3200, IBM TSO/VMS et VM/CMS, IBM PC/XT/AT, Macintosh.

Maxim est d'ores et dejà utilisé dans le domaine de la recherche, de l'industrie et de l'enseignement.

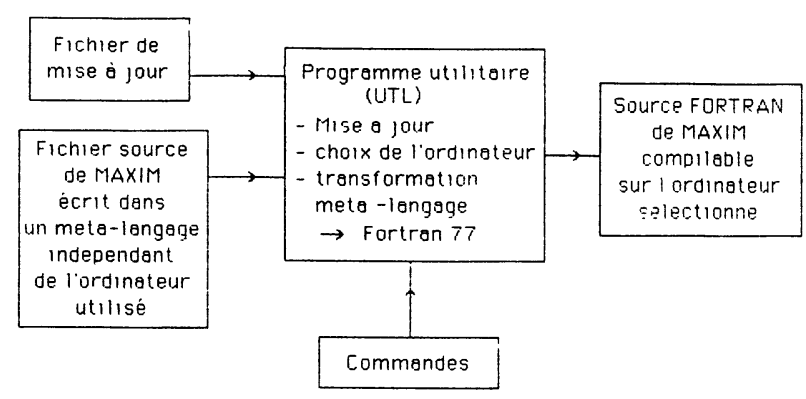

Fig. 3. - Procédure de génération automatique de MAXIM.

[MAXIM automatic generation procedure.]

\section{Facilités d'utilisation.}

4.1 Processeur D'entrée. - Les commandes utilisées pour définir un circuit sont introduites soit par l'utilisateur soit par l'intermédiaire d'un fichier de données. Ces commandes sont traitées par un analyseur lexicographique qui crée la structure de données qui sera traitée par le processeur d'initialisation et par le processeur d'analyse de MAXIM.

4.2 Processeur De SORTIE. - Une librairie graphique adaptée à différents types de terminaux permet la visualisation des formes d'ondes du circuit en fonction du temps ainsi que de quelques fonctions simples $\left(V_{i}=f\left(V_{j}\right), V_{j} * V_{j}, \ldots\right)$. Le choix du type de terminal se fait au moyen d'une commande de MAXIM.

Actuellement, les différents terminaux utilisables sont : TEKTRONIX 401X/410X, DEC VT125/240, carte graphique IBM CGA et les consoles alphanumériques.

4.3 UN LOGICIEL ÉVOLUTIF. - MAXIM était initialement dédié à la simulation de circuits logiques GaAs LSI. Mais sa structure de données interne facilement modifiable a permis l'introduction de nouvelles technologies. Il est possible d'utiliser les librairies suivantes :

- une librairie de composants (Fig. 4): MESFET's GaAs, MOSFET's Si, bipolaires homojonction et hétérojonction, diodes, résistances, condensateurs ;

- une librairie de macromodèles (Fig. 5) : circuits logiques GaAs (BFL, DCFL, SDFL), circuits logiques Si (NMOS, PMOS, CMOS), ECL, amplificateur idéal.

De nouveaux composants ou macromodèles peuvent être facilement introduits dans ces librairies grâce à la méthodologie utilisée pour les composants ou macromodèles déjà existants.

\section{Résultats et performances.}

5.1 Simulation d'Une AlU 8 Bits. - La simulation d'une ALU 8 bits est typiquement une simulation difficile (et souvent impossible) avec un simulateur électrique classique.

Le circuit simulé a une structure classique : deux ALU de 4 bits (Fig. 6b) en cascade (Fig. 6a). Les fonctions logiques sont réalisées avec des portes BFL constituées de MESFET's GaAs.

La totalité du circuit contient 120 portes et 22 entrées, soit 696 transistors et 324 diodes.

Les formes d'onde résultats de la simulation présentée figure $6 \mathrm{c}$ correspondent à l'addition binaire :

11111111

$\frac{+00000001}{100000000}$.

Le temps de calcul nécessaire à la simulation de cette addition est de $48 \mathrm{~s}$ sur un SPERRY 1100/90, de 


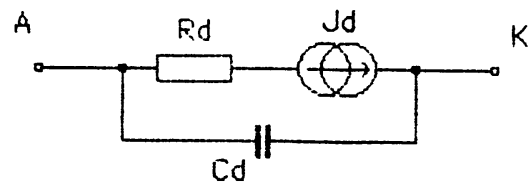

(a)

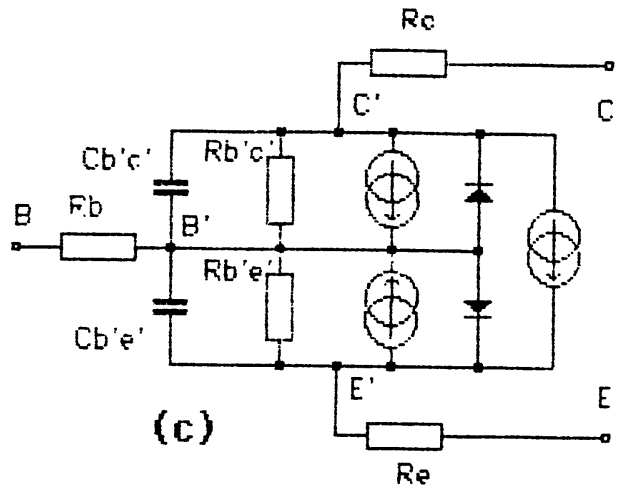

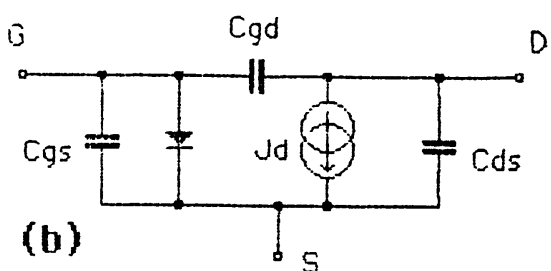

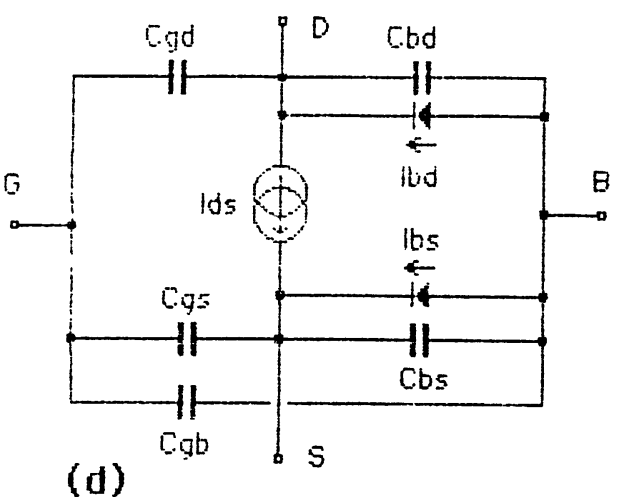

Fig. 4. - Schéma équivalent de : (a) diode Schottky, (b) MESFET, (c) BJT, (d) MOSFET.

[Equivalent circuit of : (a) diode Schottky, (b) MESFET, (c) BJT, (d) MOSFET.]
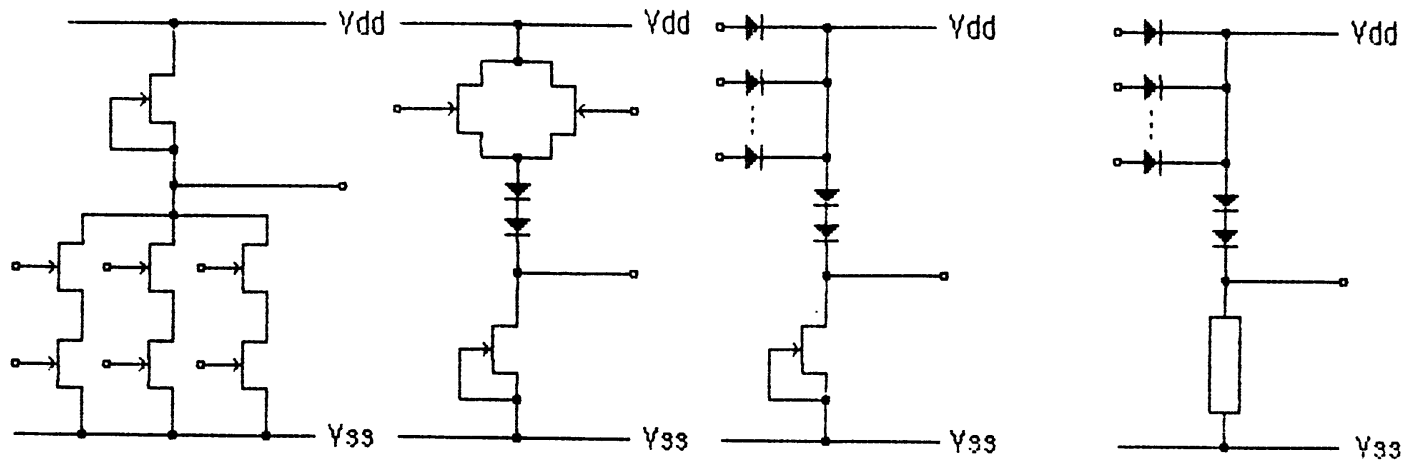

BFL/DCFL charge active

SDFL charge active SDFL charge passive
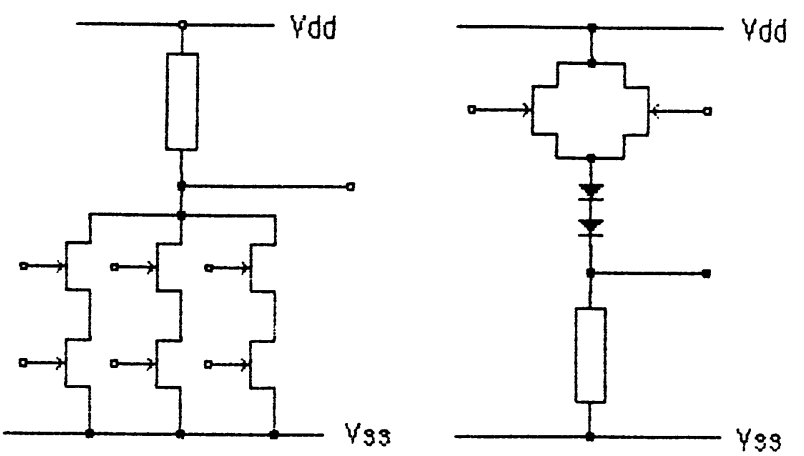

BFL/DCFL charge passive

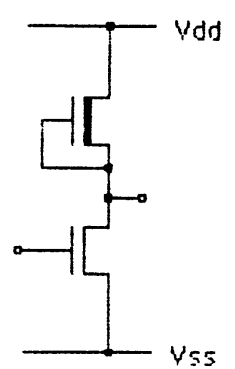

NMOS

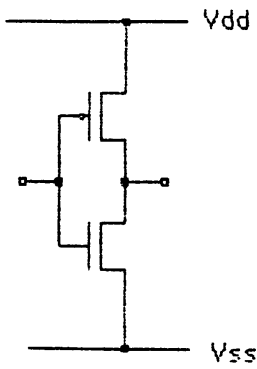

C. MOS

Fig. 5. - Macromodèles logiques dans MAXIM.

[Macromodel logic blocks in MAXIM.] 


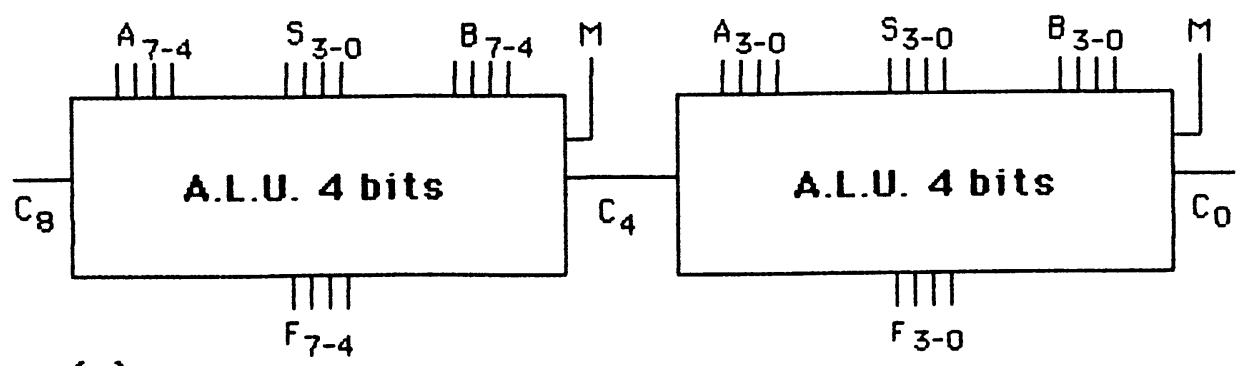

(a)
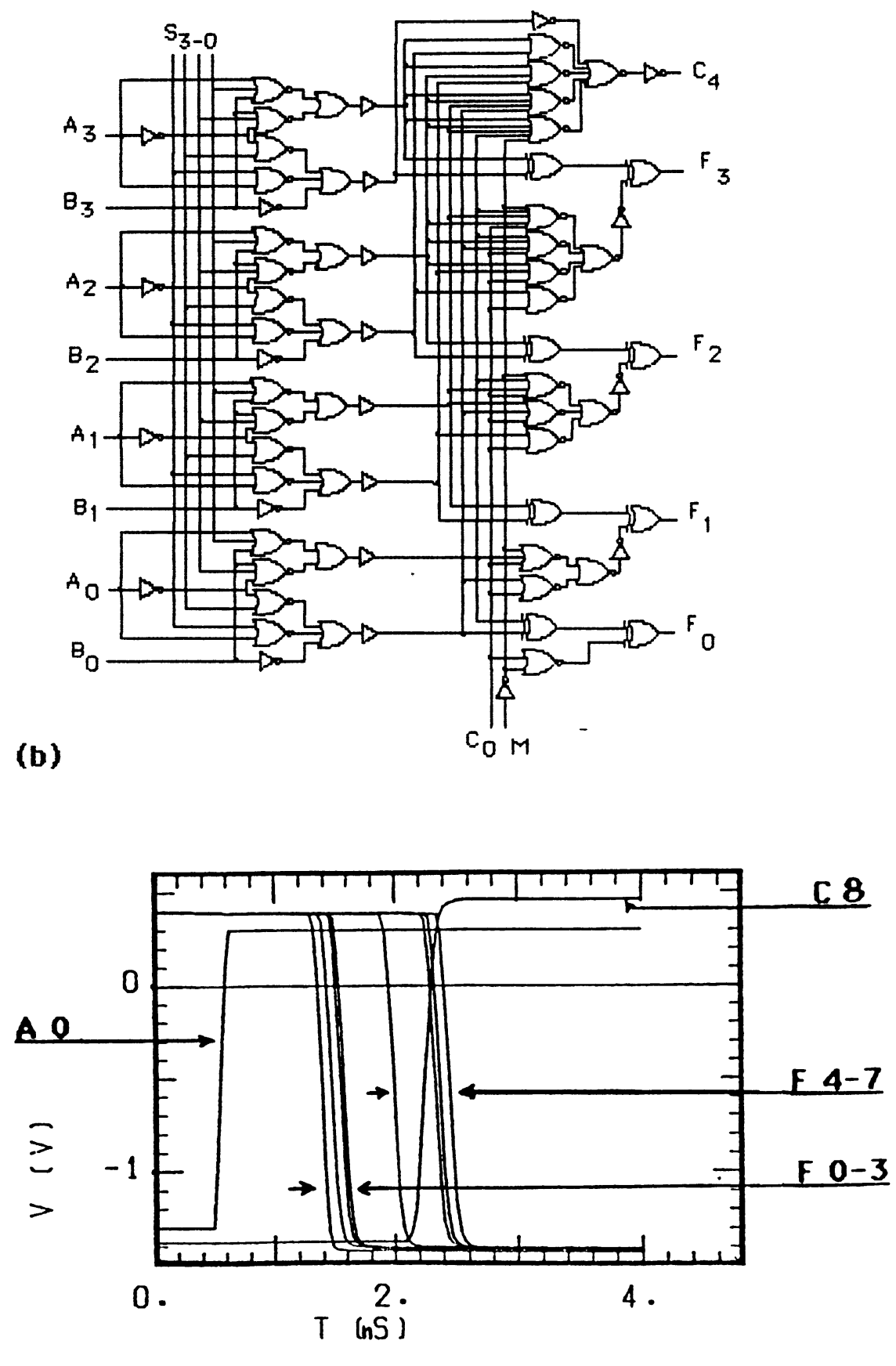

(c)

Fig. 6. - (a) unité arithmétique et logique 8 bits, (b) unité arithmétique et logique 4 bits, (c) résultat de simulation d'une addition.

[(a) 8 bits arithmetic and logic unit, (b) 4 bits arithmetic and logic unit, (c) addition simulation result.] 


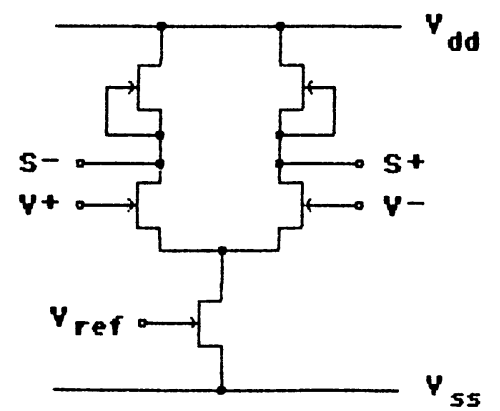

$$
\begin{aligned}
& V d d=5 \vee \quad V s s=-2,5 V \\
& \text { Vref }=-2,5 \mathrm{~V} \\
& 1: v+0,1 v 100 \mathrm{MHz} \\
& v-o v \\
& 2: V+0,1 \mathrm{~V} 100 \mathrm{MHz} \\
& V-0,03 \vee 300 \mathrm{MHZ}
\end{aligned}
$$
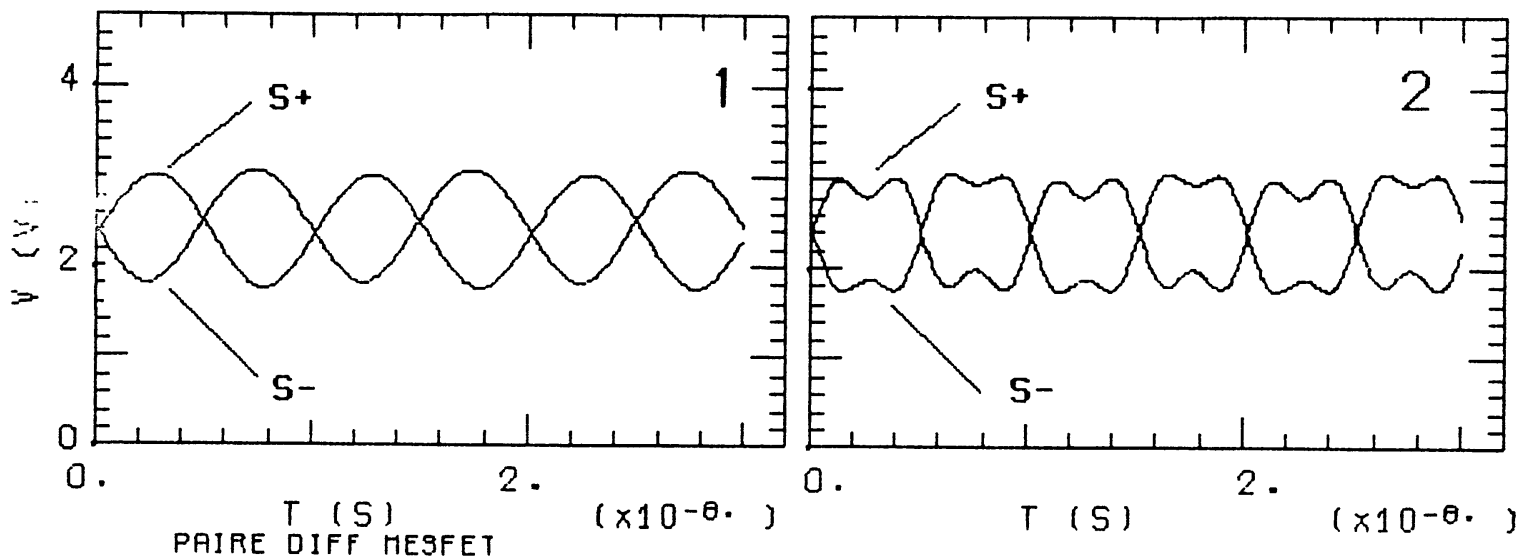

Fig. 7. - Paire différentielle MESFET's GaAs.

[GaAs MESFET's differential pair.]
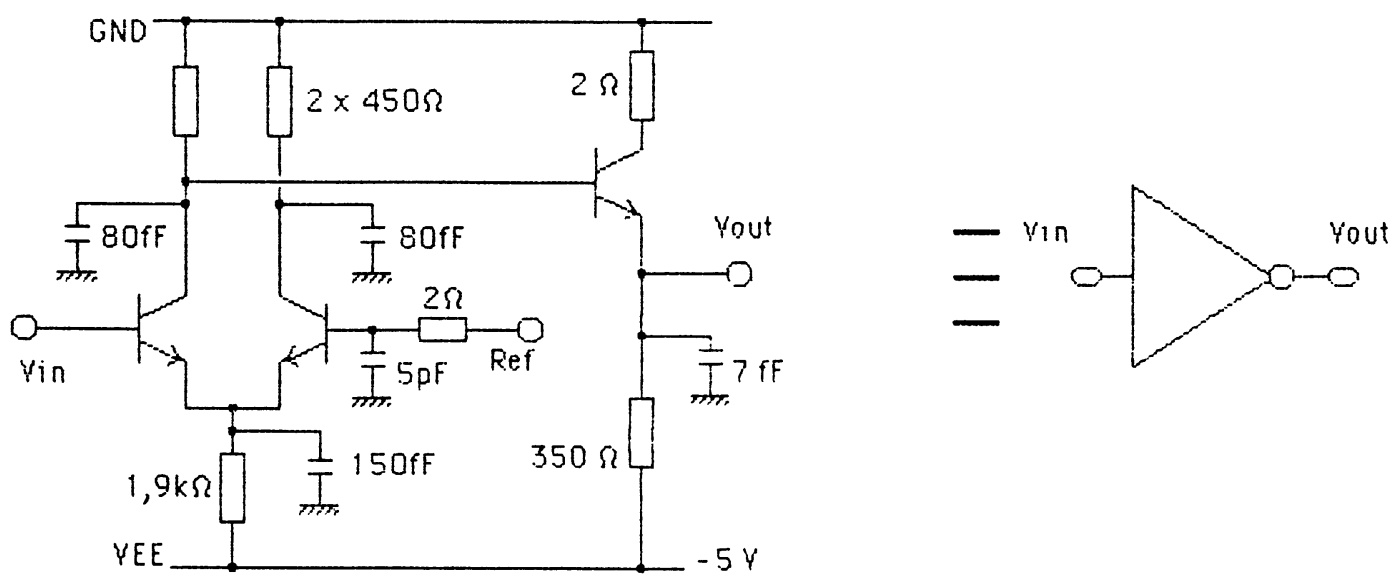

Simulation MAXIM

Simulation ASTEC

même modèle de TBH

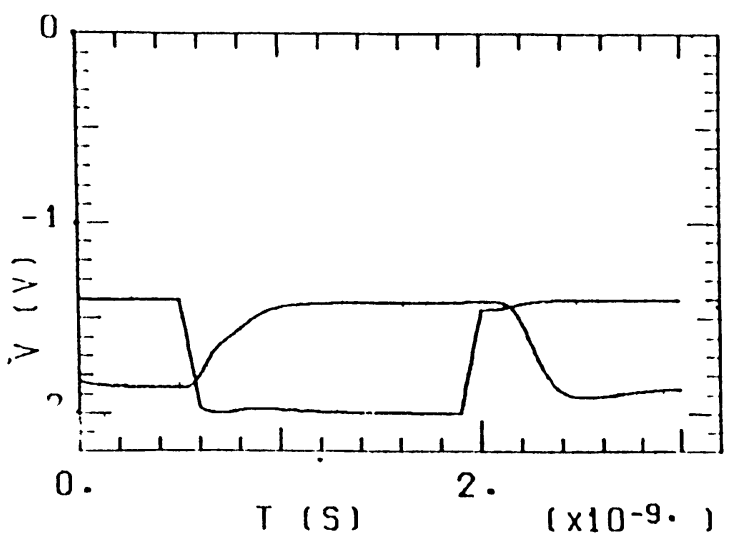

Fig. 8. - Simulation d'une porte ECL.

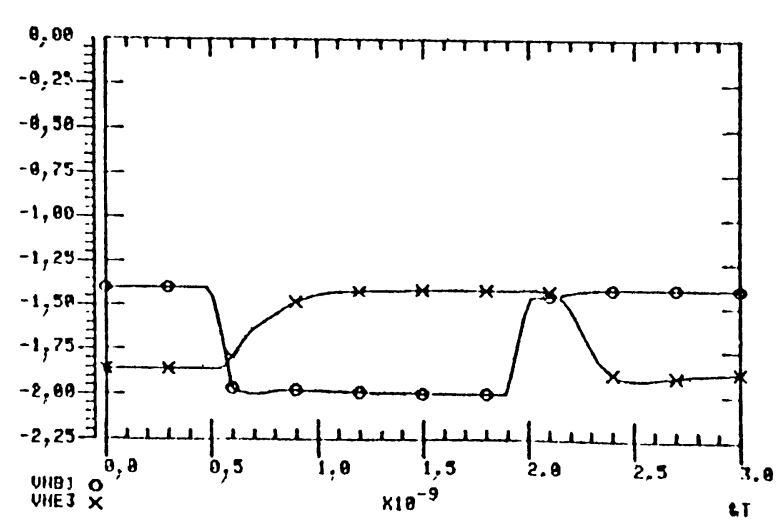

[Simulation of a ECL gate.] 
520 s sur DEC VAX 780 et de 45 min sur un PC/XT, soit respectivement $150 \mu \mathrm{s}, 2 \mathrm{~ms}$ et $10 \mathrm{~ms}$ par macromodèle et par itération. La place mémoire utilisée est $200 \mathrm{k}$ octets.

Paire différentielle en technologie MES FET GaAs.

Cette simulation montre les possibilités de MAXIM à simuler des circuits fonctionnant en régime linéaire, et aussi les possibilités de simulation en mode mixte car pour cette simulation, le composant élémentaire est le transistor; on peut donc remarquer que le critère d'unidirectionnalité entre blocs fonctionnels n'est pas respecté en certains nœuds du circuit mais que cela ne gène en rien le bon fonctionnement du simulateur (Fig. 7).

\subsection{Circuit ECL EN TEChNologie Bipolaire HÉTÉROJONCTION.}

5.2.1 Inverseur E.C.L. - L'opérateur OR/NOR E.C.L. représenté figure 8 , présente une structure classique : paire différentielle, et émetteur suiveur qui assure la sortance.

Une entrée de la paire différentielle est réservée à

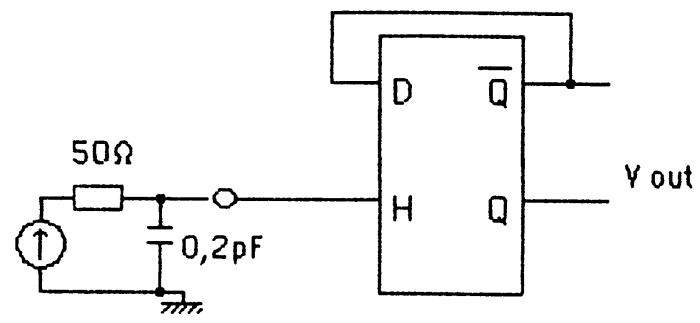

Fréquence 500Mhz

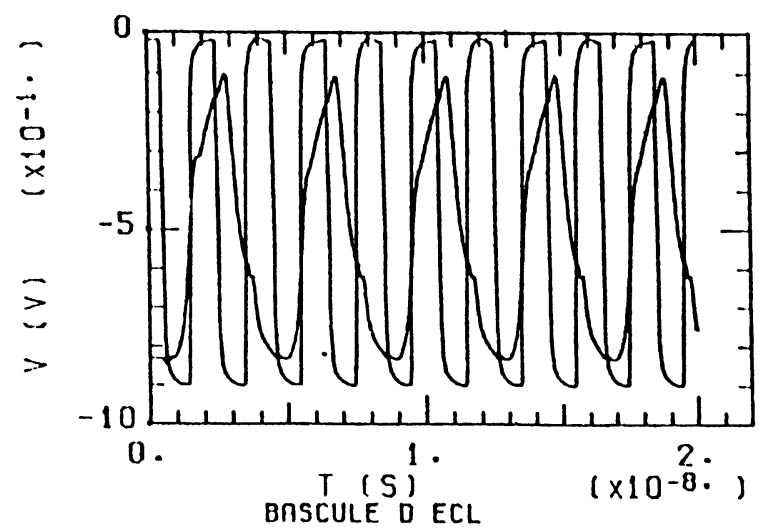

Fig. 9. - Simulation d'un diviseur par 2 CML. une source de tension de référence, les autres servent aux entrées logiques. La fonction OR/NOR à $N$ entrées est réalisée en connectant $N$ transistors identiques en parallèle.

L'excursion logique va de $-1,85 \mathrm{~V}$ à $-1,4 \mathrm{~V}$, la tension de référence est de $-1,7 \mathrm{~V}$.

5.2.2 Bascule D connectée en diviseur par deux. La porte de base est en logique C.M.L., les transistors sont soit bloqués soit passants (et non saturés). L'absence d'étage de sortie pénalise l'aptitude de la porte à la charge, mais les niveaux étant compatibles, la mise en cascade est possible.

Les états logiques sont $0 \mathrm{~V}$ pour $« 1 »,-0,8 \mathrm{~V}$ pour $« 0 »$, la tension de référence est de $-0,45 \mathrm{~V}$.

Le schéma du diviseur est rappelé figure 9 , le signal d'entrée est une source impulsionnelle. Ce circuit comporte 19 transistors.

La réponse du circuit est représentée à la limite de fonctionnement du diviseur :

$\rightarrow f=500 \mathrm{Mhz}$, les signaux sont très dégradés mais la division s'effectue encore.

$\rightarrow f=555 \mathrm{MHz}$, le diviseur « décroche » après un léger glissement qui accentue un aléa lors de la descente du signal de sortie.

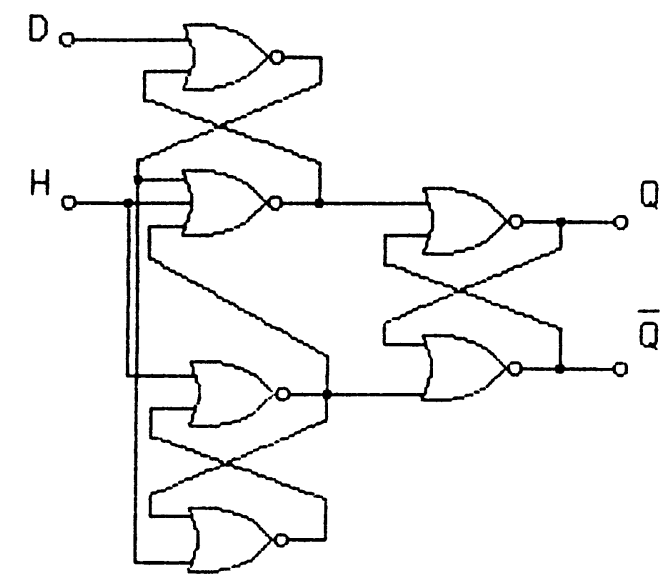

Fréquence 550Mhz

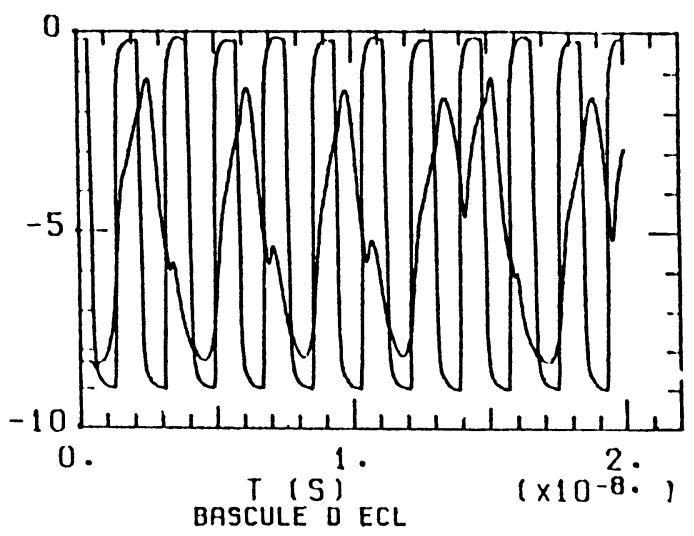

[Simulation of a CML divider.] 

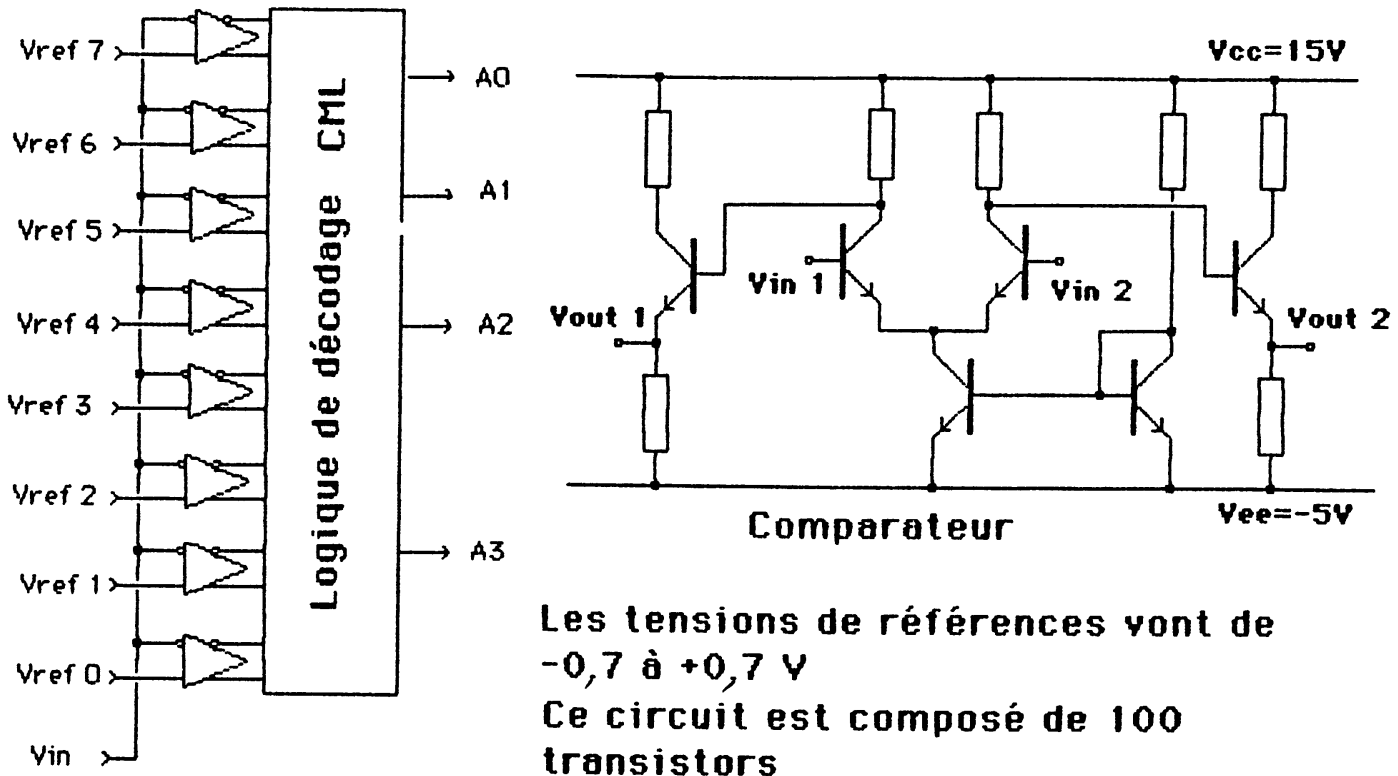

Les tensions de références yont de $-0,7$ ò $+0,7 \mathrm{~V}$

Ce circuit est composé de 100 transistors

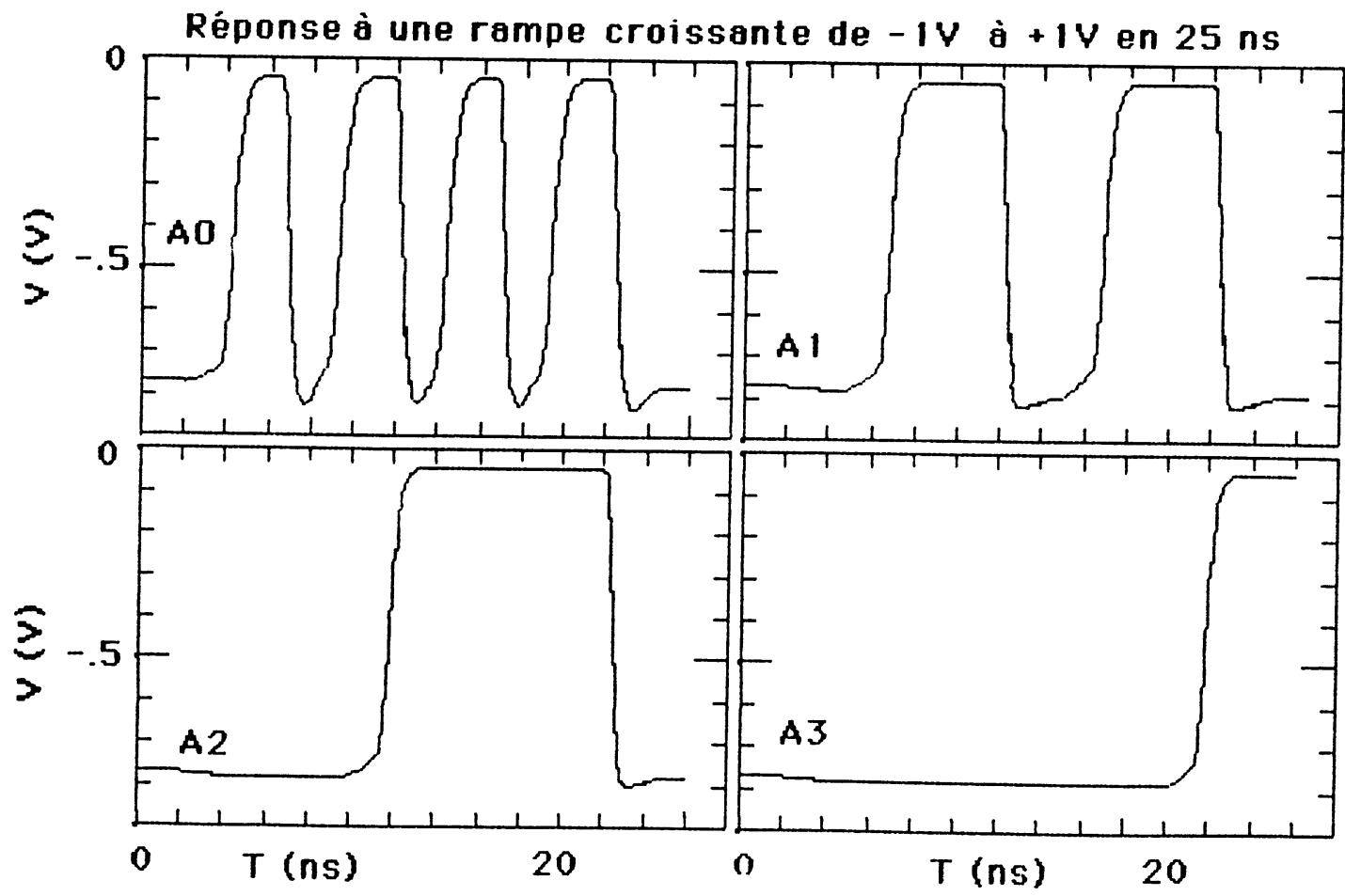

Fig. 10. - CAN type « flash».

5.2.3 Convertisseur Analogique Numérique type "flash» 3 bits. - Ce circuit est constitué d'une série de comparateurs qui comparent la tension à convertir à des tensions de référence (le générateur de tension de référence n'est pas simulé), ces comparateurs sont constitués d'une paire différentielle, suivie d'un étage de mise à niveau CML qui commande la logique de décodage (Fig. 10).

Ce circuit comporte 100 transistors, le temps de simulation sur SPERRY 1100/90 est de $300 \mathrm{~s}$ (soit environ $200 \mu$ s par macromodèle, par itération).

Le signal appliqué au circuit est une rampe
[Flash DAC.]

croissante en $25 \mathrm{~ns}$. Les sorties évoluent alors en comptage selon le code binaire classique.

\section{Conclusion.}

MAXIM est un macrosimulateur électrique développé pour la simulation rapide de circuits complexes. Sa souplesse d'utilisation, sa structure de données très adaptative lui ont permis de s'adapter sans difficultés à des technologies et à des circuits nouveaux.

Du fait des temps de calcul relativement faibles 
requis par MAXIM, la voie lui est tout à fait ouverte à la simulation de circuits complexes mélangeant logique et analogique comme par exemple des convertisseurs analogiques numériques.

\section{Bibliographie}

[1] Pone, J.-F., Murzin, J.-Y., Dusausay, S., PhilipPe, P., CASTAGNE, R., MAXIM, an electrical macrosimulator for GaAs MSI and LSI logic circuits. Proceeding of the NASECODE IV conference 19-21 June 1985, Dublin (Boole press).

[2] Voir l'article de P. CrozAt dans ce numéro.

[3] GEAR, C. W., Numerical initial value problems in ordinary differential equations (Prentice-Hall, Inc Englewood Cliffs) 1971.

[4] SenN, P., Hennion, B., Eldo, un nouveau simulateur électrique pour VLSI, suivi d'exemples sur les circuits à capacités commutées. Actes de la conférence sur les circuits analogiques, Paris, Octobre 1984.

[5] Etiemble, D., Houssenbay, M., $A$ third generation circuit simulator running on IBM-PC. Université P. et M. Curie, rapport MASI, Paris, Octobre 1986.

[6] NAGEL, W., SPICE 2, a computer program to simulate semiconductor circuits. University of California, Berkeley, Memo No. ERL-M520, May 1985.

[7] HeydemanN, ASTEC 3, Manuel de référence. Compagnie Internationale de Service en Informatique, Juillet 1985 . 